cardiac cerebrovasular events (MACCE, including cardiac death, non-fatal myocardial infarction, revascularisation and stroke) and readmission for cardiovascular events were assessed. Survival curves were performed using the Kaplan-Meier method with the log-rank test. The association between obesity and clinical outcomes was future examined using univariate and multivariate Cox proportional hazard models.

Results The median duration of follow-up was 367.34 \pm 109.00 days. Obesity was associated with younger age $(p<0.001)$, a higher prevalence of male $(p<0.001)$, hypertension and hyperlipidaemia (both $p=0.001)$; the higher levels of low density lipoprotein $(p=0.01)$, cholesterol $(p=0.001)$ and triglycerides $(p<0.001)$. PCI characteristics and treatments were similar across BMI category. At follow-up, MACCE/ readmission for cardiovascular events free survival rate was not significantly different among three groups; after adjustment confounders, obese was the independent risk factor for cardiac death $(p=0.04)$, and non-fatal myocardial infarction $(p=0.04)$.

Conclusions Patients with obesity present with STEMI having urgent PCI have the MACCE/readmission for cardiovascular events free survival rate similarities to normal weight and overweight counterparts at follow-up. Obesity was independently associated with the higher incidence of the cardiac death and non-fatal myocardial infarction. The 'obesity paradox' was not observed in outcomes.

\section{GW23-e0874 IMPACT OF OBESITY ON OUTCOMES OF CHINESE PATIENTS WITH ST-SEGMENT MYOCARDIAL INFARCTION HAVING URGENT PERCUTANEOUS CORONARY INTERVENTION}

doi:10.1136/heartjnl-2012-302920d.8

${ }^{1}$ Sunyujiao, ${ }^{2}$ Jiangdaming, ${ }^{3}$ Zhangbo, ${ }^{1} Y u h a i j i e,{ }^{1}$ Gaoyuan, ${ }^{1}$ Liyuze, ${ }^{1}$ Oiguoxian. ${ }^{1}$ The First Affiliated Hospital of China Medical University; ${ }^{2}$ Central Hospital DanDong; ${ }^{3}$ First Affiliated Hospital of DaLian Medical University

Objectives The study analysed the impact of obesity on outcomes of Chinese patients with ST-segment myocardial infarction (STEMI) having urgent percutaneous coronary intervention (PCI).

Methods 421 patients with STEMI having urgent PCI who were admitted to the 20 hospitals in LiaoNing region from 2009 until 2010 were enrolled. Patients were stratified according to Body Mass Index (BMI) as normal weight $\left(18.5 \mathrm{~kg} / \mathrm{m}^{2} \leq \mathrm{BMI}<24.0 \mathrm{~kg} / \mathrm{m}^{2}\right.$, $\mathrm{n}=149)$, overweight $\left(24.0 \mathrm{~kg} / \mathrm{m}^{2} \leq \mathrm{BMI}<28.0 \mathrm{~kg} / \mathrm{m}^{2}, \mathrm{n}=196\right)$, or obese (BMI $\geq 28.0 \mathrm{~kg} / \mathrm{m}^{2}, \mathrm{n}=76$ ). At follow-up the main adverse 